\title{
Brief Talk about Creative Design of Interior Space Environment
}

\author{
Sheng Zhang \\ Jiangxi Institute of Fashion Technology, Nanchang, 330201, China
}

\begin{abstract}
Keywords: Interior space. Environmental design. Creative ways
\end{abstract}
\begin{abstract}
With the continuous improvement of people's living standards, the requirements of the interior environment have changed. In modern society, people think that the interior environment should have creativity and artistry. The interior design has always been an important part of the decoration. Hence, how to design the interior space with artistry instead of normal is key element to designers for a long time. Based on above-mentioned background, this article briefly discusses the connotative meaning of creative design of interior space circumstance, analyzes several methods of creative design and studies differentiated creative design in different interior space. Meanwhile, it also discusses application of creative design from two aspects of interior furnishing and space planning. All these activities aim at optimize design of space environment and make the idea into design.
\end{abstract}

\section{Introduction}

Interior design is a reconstruction activity for the purpose of comfortable living and using function, of which the main object is building structure through scientific planning and related facilities as auxiliary materials as well as study of natural environment and historic culture. The interior design generally starts aesthetic as point for interior furnishings, interior space transformation. However, traditional interior design has been unable to meet the requirements of the people on interior space. In modern society, artistic style can be fully reflected through more elegant, comfortable and high utilization of space environment which could be designed by various design methods and ideas. Good ideas can make the design results fully reflect the characteristics of the interior environment during interior space design, while based on user requirements will be designed to be more humanized. The interior environment can bring the user to certain spacial scale that can help them to find "soul" of the environment to some extent. Space design has become one of the main aspects of the design under the increasing demands on indoor environment by people. Without creativity there would be no development, which refers to creative design is the power of designers' long-term study and practice. For these reasons, the article puts forward some direction for reasonable creative design of environmental art with interior space design as the main line and creative design as standard.

\section{The Representing Methods of Creative Design in Interior Environment}

\section{Green Design}

Green design refers to environmental design far from green in interior environment. As natural environment gradually becomes bad, interior design shall focus on environmental protection to reduce energy consumption and pollution level. Green design as a tendency in current design, people give more attention on interior design.

The material for green design is mostly energy conservation and environmental protection, such as exterior wall thermal insulation material and portable board for adjustable temperature and others that can reduce usage of indoor air conditioning. The usage of air conditioning can cause bad effect to atmosphere and consume large amount of energy, which is not conducive to creation of resource-saving and environment-friendly society. Furthermore, we can install some board on the wall that can place some flower to improve the green atmosphere of whole space or made some modern art forms on the wall through painting, wall paper or glass tiles that can break up the monotony. 


\section{Change Pattern to Create a Natural Ecological Space}

Different from green design, ecological space implements ideas from the perspective of spatial pattern. Hence, the designer needs to recognize interior environment and break the limit of the outdoor and indoor space through time movement process which can integrate outdoor elements into indoor environment or let indoor space become part of outdoor space.

Change this pattern, of course, should also be based on the actual situation of the interior space. If not, don't have to insist it. We can make the interior space look more ecologicalization through place furnishings with native culture or resource characteristics.

\section{Ingenious Application of Light}

Due to human visual illusion, designers can use light to create changes of interior space. Specifically, light intensity, projection form, shadow and differences of the light source will cause the user to feel diversification of indoor space. That is to say, we can integrate floor-lamp with ceiling lamp to form differences of the light source and changes of light and dark in space.

Through ingenious application of light, the user will have aesthetic feeling from space and enjoy the novelty on the vision. However, due to the introduction of the concept of energy saving design, the use of light could not simply confined to artificial light; it also should integrate natural light appropriately. Doing so can make light fully played. At this point, China Pavilion of World Expo and partial Olympic Arena adopt this method to use nature light, integrating with artificial light to create a beautiful scene and enhance the appeal of the interior space.

\section{Organic Space Environment Design}

In modern society, art environmental design should use more modern residential facilities to improve indoor environmental quality through high-tech innovations, using user's psychology and visual patterns. Designers should carefully study the psychological state of the user as well as the direction of environment on the psychological state; study that whether organic space impacts on user psychological state.

From many years of experience, the people between 20-40 years old like the organic space design and have high degree of reliance on science and technology products. Environmental psychology mentioned that there is an interaction between space environment and users, which has mental stimulation to the users by form, scale, constituent part of space environment. The users' response to such stimuli can be called environmental stimulation effect, which related to quality of the artistic environment.

\section{Creative Design Points for Different Interior Space}

\section{A close-type space}

A close-type space refers to use the material with strong isolation in space, including wall other than screen and other similar products. In this close-type space, the isolation will affect feeling, hearing and vision of people. At present, most common close-type space belongs to residence building which is rectangular or square pattern with thick and opaque material as isolation materials and keeps independent state in space.

This close-type space will give the user a quiet, solemn feeling, which is suitable for academic researchers or introvert. Artistic environment in this space design should be given priority to white as main color instead of exaggerated elements. In short, the close-type space design shall be brought privacy and security to users.

\section{A open-type space}

Such interior space belongs to open-type space that can connect with surrounding environment. For example, there is a balcony that can be seen the outside view directly. If you want to achieve spatial integration effect, such design is best choice that can link the indoor space with outdoor space by borrowed scenery and opposite scenery.

A open-type space has an advantage of openness in artistic environment design. Such space will give you a lively feeling relative to the closed space. However, one thing to note is that the space design will be depended on degree of opening, enclosure and on-off control. Designers can use 
transparent glass wall instead of wall, then matching a curtain with similar color of outside view. When night comes, you can put down the curtain that makes the space closed. When day comes, you can pull back the curtain and see the landscape. Hence, this design can achieve space circulation effect. With the help of transparent glass wall, visual space is magnified. In addition, the creative design of metope brings certain sense of flow to enrich content of space.

\section{A flow-type space}

There is less flow-type space in dwelling building, mostly are public buildings. The space constantly changes as transfer of sight and flow condition. In other words, there is continuity among spaces. This design can achieve space "dialogue" effect.

The design for flow-type space shall follow the word "flow". That is to say, the design is not only connect with surrounding environment, also penetrate into each other. We should look space as object with vitality rather than negative, stationary objects. Creative design should keep reasonable transition during flow-type space. Making space full status can be through improvement of artistry and skill. For instance, if two spaces are simply connected, the communication between these two spaces is a bit thin. Curve design can make the plane with some sense of flow. That is to say, the stairs, metope and other rigid objects can be designed to arc-shaped and streamlined shape which enhance the connection between spaces and brings fashion and dynamic feel. We can adopt this flow space art design form in environmental design of restaurant that can let customers feel dynamism of the restaurant, avoiding the depressing feeling.

\section{A virtual-type space}

Virtual space, also called imaginary space, belongs to a form that has certain range with intangible interface. In other words, this is an imaginary space, which can be referred to as psychological space. This environment design has no fixed isolated form with poor limited degree, which means that the spatial planning all carried out by user imagination and visual gestalt. Therefore, this creative design has strong subjectivity and high variability.

In virtual-type space, objects with limited are furniture, stand column, partition and others instead of walls. Hence, this can build difference imagination space from psychological feelings and visual perception of users. In this design pattern, space environment art design was endowed with diversified colors that could provide reference element for decorate. In a large space, the rational use of virtual space allows users to partition into several small spaces psychologically which can be designed by their favorite style.

\section{Innovative Direction of Interior Space}

\section{Furnishings}

Design effect is largely affected by interior furnishings during art space design. Furnishings with different functions and style also can be able to become important point of interior design to achieve unique effect. In the selection of furnishings, you should first make sure that designers have a good idea and theme to avoid fuzzy indoor theme caused by cluttered display.

It should be noted that the design of furnishings should be established on the basis of spatial planning, which refers to you shall select appropriate style furnishings according to spatial layout style. Meanwhile, the material of furnishings also affects artistic effect. In traditional design, people's understanding of interior furnishings is limited to functional products, such as screen and tea table, who rarely notice small interior decoration items. With gradually improvement of living standard and life sentiments, interior furnishings has changed from the traditional large pieces of furniture to small items like metope aquarium, indoor incense and others. However, small items shall be placed in the light of residential space design and overall style design. For selection of furnishings, users can work together with designer that can fully express design idea in best results.

\section{Spatial Planning}

Before designing, interior space is a complete space. The designer shall invest time and effort to divide such space that can be achieved effective design through rational distribution. Moreover, it has innovation in space design. The space will be divided by function in traditional space design. For 
example, a large space will be divided into several small spaces like master bedroom, guest bedroom, living room, study, kitchen, etc.. But the weakness is that the space will be looked a bit dull, while difficult to complete the design innovation.

Space design is an aesthetic design, while furnishings, decoration design shall be consistent with such design. For human atmosphere, it also should be based on such space. In other words, designers shall fully understand space function so as to show its innovative. In terms of current space design, the common means of interior design includes space-partitioning, space-separation, space variation, partition, bright, well-proportioned, borrowed scenery and space level processing. In terms of innovation, the designers focus attention on more humanized space design other than space with dull and limited, which will be achieved through reconstruction of space with fold and enlarge.

The designers will use different design languages in carrying out space artistic designing, while the spatial effects are different too. In terms of creativity, everyone's understanding of creativity will have differentiation that caused by education, quality of life, ages, marital status of users and other factors.

\section{Conclusions}

With the continuous improvement of people's living standards, tastes, needs and money, the requirements on living condition also increase. People are not only concerned about comfort of the traditional interior decoration, but transfer the attention to interior design connotation, space usage and aesthetic design usage and so on. On the whole, living utilitarian function combined with people-oriented design aesthetics shows concordance which reflects material living standards and mental attitude of resident in new era. Therefore, the designers shall absorb more design concept and aesthetic thought in future interior space design to meet people's growing material and spiritual needs through continuous exploration and integration.

\section{References}

[1] Zhang Xiaohua: Explore Creative Design of Interior Space [J]. Art Criticism, 2009 (08)

[2] Xi Xiaoxi, Du Wenzhang, Li Jingjuan: Color Analysis of Interior Space Design [J]. Dawutai, 2012 (08).

[3] Wang Lin: Talking About Lighting Design of Interior Design [J]. Art Education Research, 2013 (02).

[4] Zhao Wei: Ceramic Decorative Elements in Interior Design Space [J]. Yihai, 2013 (06).

[5] Wang Haifan: Analysis of Light Environment Design in Interior Design [J]. Journal of Changzhou Institute of Technology, 2011 (08).

[6] Lu Dai Ling: Important Function of Creativity in Interior Design [J]. Yihai, 2010 (08). 